Journal of Computer Science 5 (1): 57-63, 2009

ISSN 1549-3636

(C) 2009 Science Publications

\title{
A Channel Adaptive Energy Efficient and Fair Scheduling Media Access Control Protocol for Mobile AdHoc Networks
}

\author{
P. Priakanth and P. Thangaraj \\ Department of Computer Science and Engineering, \\ Kongu Engineering College, Perundurai-638052, Erode, Tamilnadu, India
}

\begin{abstract}
Problem statement: A major drawback in the existing protocols in dealing with energy management issues is that the time varying nature of the wireless channels among the ad hoc nodes is ignored. Approach: This study proposed a channel adaptive energy efficient Medium Access Control (MAC) protocol, for efficient packets scheduling and queuing in an ad hoc network, with time varying characteristic of wireless channel taken into consideration. Every node in the proposed scheme estimates the channel and link quality for each contending flow based on which a weight value is calculated and propagated using the routing protocol. Since a wireless link with worse channel quality can result in more energy expenditure, the transmission was allowed only for those flows whose weight is greater than Channel Quality Threshold (CQT). For flows with weight less than CQT, the packets were buffered until the channel and link quality recovers or the weight becomes greater than CQT. To avoid buffer overflow and achieve fairness for the poor quality nodes, a fair scheduling and queuing algorithm is designed where in the CQT is adaptively adjusted on basis of the current incoming traffic load. Results: Simulation results showed that the proposed MAC protocol achieves substantial energy savings with better fairness and increased throughput. Conclusion: The designed protocol provided an efficient packets scheduling and queuing in an ad hoc network, with time varying characteristic of wireless channel taken into consideration.
\end{abstract}

Key words: AdHoc networks, MAC protocol, scheduling, energy saving, bandwidth

\section{INTRODUCTION}

In recent years, more attention has been given to ad-hoc wireless networking, enabling many interesting usage scenarios however several challenges are being posed. Conventionally, cellular telephony and Internet connectivity via radio modems applied wireless networking. The fixed, wired base station was provided with single hop connectivity by these systems. The formation of multi-hop networks without predetermined network topologies is attempted by the adhoc wireless network systems.

The interaction between the nodes is in peer-topeer form, whereas the nodes communicate with a centralized base station in case of cellular networks. Dynamically changing topologies, which is direct result of the mobility of the nodes, characterize the ad-hoc networks. Many advantages can be offered by such systems, which do not rely on extensive and expensive installations of fixed base stations throughout the usage area. They can perform route selection when multiple routes to the same node or base station are available, on the basis of various metrics such as robustness and energy cost. Instead of using a distant, intermediate base station for communication, nodes can communicate directly with each other when possible, thereby helping to conserve energy and improving throughput. Various applications, ranging from the monitoring of herds of animals to supporting communication in military battlefields and civilian disaster recovery scenarios are enabled by these systems.

The mobility of nodes and its deployment with little network planning is necessitated by many of these applications. The size is limited by the mobility of the nodes; in turn the energy reserves available to the nodes are limited.

Therefore, the design of ad-hoc networks necessitates energy conservation as a key requirement. The ignorance of time varying nature of the wireless channels among the ad-hoc nodes by the existing protocols is one major drawback when dealing with energy management problem ${ }^{[1]}$. In hostile deployment circumstances, either increasing the transmission power

Corresponding Author: P. Priakanth, Department of Computer Science and Engineering, Kongu Engineering College, Erode, Tamilnadu, India 


\section{J. Computer Sci., 5 (1): 57-63, 2009}

level or adding Forward Error Correction (FEC) to the raw data can reinforce reliability of data transmission. Since the channel quality changes with time, the amount of incorporated error protection should also vary with the instantaneous channel condition to make sure the Bit Error Rate (BER) is above the required level. Consequently, the poorer the wireless link, the more amount of error protection is redundant in the transmitted packet and vice versa.

Our contribution: In this study, we design a channel adaptive energy efficient MAC protocol considering the time varying characteristic of wireless channel, for efficient packet scheduling and queuing in an ad-hoc network. We propose a network system to implement this basic idea in the real situation, where every node can decide the state of its communication component with respect to the current link condition.

Review of prior works: Padhye et al..$^{[5]}$ have proposed a simple, empirical methodology to estimate pairwise interference using only $\mathrm{O}\left(\mathrm{n}^{2}\right)$ measurements. They showed that their methodology accurately predicts pairwise interference among links in their test bed in a variety of settings. They hope to increase the accuracy of their methodology by accounting for the impact of four factors.

Ming et $a l .^{[6]}$ have demonstrated the effects of unfairness performance of the IEEE 802.11 standard under a pre-computed routing scheme for two network configurations and a fixed traffic patterns. They have examined the send rate, a quantity similar to the local throughput, for very simple chain and lattice networks with regular traffic patterns and then they have investigated the performance of both types of fairness improvement schemes on this rate.

Kone et $a l .{ }^{[7]}$ have developed QUORUM, a novel QoS-aware routing protocol for wireless mesh networks. Specifically, QUORUM takes three QoS metrics into account: bandwidth, end to end delay and route robustness. To optimize QUORUM for wireless mesh networks, they proposed several mechanisms for topology aware route discovery that drastically reduced the control overhead and network congestion from route discovery. In addition, they introduced the novel DUMMY-RREP data latency estimator and show it to be effective in providing accurate estimates of end-toend delay experienced by data packets. Finally, their proposed link robustness metric allows QUORUM to punish and discourage free-riding behavior by selfish nodes.

VO and Hong ${ }^{[8]}$ have proposed a novel approach to control the traffic to avoid congestion by decoupling forwarding process from the routing process. The proposal is interested in provisioning quality of service guarantees for planned Wireless Mesh Networks which are being used widely as a broadband wireless access network.

Cheng et al. ${ }^{[9]}$ have proposed MARIA (Mesh Admission control and Qos Routing with Interference Awareness), a scheme towards enhancing QoS support for multimedia in wireless mesh networks. They have implemented an on-demand routing scheme that explicitly incorporates the interference model in the route discovery process. Simulation results demonstrate that by taking interference into account MARIA outperforms the conventional approach.

Chen et al. ${ }^{[10]}$ a dynamic window concept $(\mathrm{m} ; \mathrm{k})$ is presented to record the link historical information and a link quality estimation based routing protocol (LQER) are proposed, which integrates the approach of minimum hop field and $(\mathrm{m} ; \mathrm{k})$. The performance of LQER is evaluated by extensive simulation experiments to be more energy-aware, with lower loss rate and better scalability than $\mathrm{MHFR}^{[1]}$ and $\mathrm{MCR}^{[2]}$. Thus the WSNs with LQER get longer lifetime of networks and better link quality.

Overview of our proposed MAC protocol: Packet transmission through a link of high quality consumes less energy than that needed through a "bad" link. Based on this observation, in our proposed scheme, each node should possess the ability to decide the state of its communication unit with respect to the current condition of the wireless link between it and the sink. Every node estimates the channel and link quality for each contending flow. Based on the channel and link quality, a weight value is calculated and propagated along with the data packets using the routing protocol. Since a wireless link with worse channel quality can result in more energy expenditure, the transmission is allowed only for those flows whose weight is greater than Channel Quality Threshold (CQT) value. For flows with weight less than CQT, the packets are buffered until the channel and link quality recovers or the weight becomes greater than CQT.

To avoid buffer overflow and achieve fairness for the poor quality nodes, we also design a fair scheduling and queuing algorithm. In the fair scheduling algorithm, we adaptively adjust the minimum quality threshold, based on the current incoming traffic load. For this, the buffer and queue length values of the node are continuously monitored for a specified period. Based on the queue length variations in that period, the traffic load L can be predicted. Whenever there is a buffer overflow, the threshold is adaptively adjusted, based on 
the predicted traffic load. i.e., threshold will be reduced or increased if the traffic load is increasing or decreasing, respectively. Thus, we can achieve a balance between energy efficiency and fairness.

Estimating the link quality with residual bandwidth and energy: The link quality or link metrics such as: bandwidth, delay, loss rate and more is measured by the MAC layer. Link metrics are then introduced to IP routing protocol. The link metrics are taken into account in order to calculate the path for the new incoming flow by the routing algorithm.

The standard "Hello" message is credited with the sender's current bandwidth and energy level usage as well as the sender's one-hop neighbors' current bandwidth and energy usage ${ }^{[3]}$. On the basis of the information provided in the "Hello" messages and knowledge of the frequency reuse pattern, every host estimates its residual bandwidth and energy. Thus the creation of extra control messages by using the "Hello" messages to disseminate the bandwidth and energy information is avoided. Based on the scrutiny of the packets provided into the network by the host, each host estimates its utilized bandwidth. The value at the host is recorded and updated periodically by the bandwidth utilization register. On the basis of the information provided in the "Hello" messages, every host adds its available energy to the energy of the downstream node.

On the basis of the information from hosts within two-hops, the residual bandwidth and energy information are approximated by every host. The information about the first neighboring host can be obtained directly. In order to disseminate the information about the second neighboring host, we propose using hop relay. AODV updates ${ }^{[4]}$ the neighbor caches by the employment of "Hello" messages.

The "Hello" message is modified such that two fields are included. The host address, consumed bandwidth, consumed energy, timestamp are included in the initial field and the neighbors' addresses, consumed bandwidth, consumed energy and timestamp are included in the second field. The host examines the timestamp of the "Hello" message it receives from its neighbors and concludes whether this "Hello" is a restructured one. A cache structure which includes a first neighbor table and a second neighbor table is employed. In the cache the second neighbors are linked with their corresponding first neighbors.

When the host obtains the information about the bandwidth consumption of its first neighbors and its second neighbors, it estimates the residual bandwidth $\mathrm{R}_{\mathrm{bw}}$ as:
$\mathrm{R}_{\mathrm{bw}}=\left(\mathrm{C}_{\mathrm{bw}}-\mathrm{U}_{\mathrm{bw}}\right) / \mathrm{W}_{1}$

Where:

$\mathrm{C}_{\mathrm{bw}}=$ Channel bandwidth

$\mathrm{U}_{\mathrm{bw}}=$ Used or consumed bandwidth

$\mathrm{W}_{1}=$ Weight factor

It is essential to divide the residual bandwidth with weight aspect owing to the IEEE 802.11 Mac protocol's property and the routing overhead.

Similarly the residual energy $R_{e}$ is estimated as:

$\mathrm{R}_{\mathrm{e}}=\left(\mathrm{C}_{\mathrm{e}}-\mathrm{U}_{\mathrm{e}}\right) / \mathrm{W}_{2}\left(\mathrm{C}_{\mathrm{e}}-\mathrm{U}_{\mathrm{e}}\right)$

Where:

$\mathrm{C}_{\mathrm{e}}=$ Initial energy

$\mathrm{U}_{\mathrm{e}}=$ Used or consumed energy

$\mathrm{W}_{2}=$ Weight factor

Now the score $\mathrm{S}$ can be calculated as the combined sum of residual bandwidth and energy:

$\mathrm{S}=\mathrm{R}_{\mathrm{bw}}+\mathrm{R}_{\mathrm{e}}$

Estimating the channel state: The Channel State Information (CSI) characterizes the combined effect of signal propagation factors. It is measured as Signal-toNoise Ratio (SNR) of known pilot symbols and transmitted along a feedback channel. It is assumed that the CSI remains constant for the duration of one frame. A real-time monitoring of the change of the CSI of the wireless link is necessary for a mobile node.

We can adopt a simplified tone channel as the CSI and channel state indicator for the medium access control of each node. Mathematically, the signal from this tone channel can be expressed as:

$\mathrm{T}(\mathrm{t})=\mathrm{A} \sum_{\mathrm{n}=1}^{\mathrm{K}} \mathrm{g}\left(\mathrm{t}-\mathrm{nT} \mathrm{T}_{\mathrm{P}}\right)$

Where:

$\mathrm{A}=$ The amplitude of the signal

$\mathrm{g}(\mathrm{t})=\mathrm{A}$ pulse signal with period of $\mathrm{T}_{\mathrm{p}}$ and $\mathrm{K}=\mathrm{T}_{\mathrm{s}} /\left(\mathrm{T}_{\mathrm{a}}+\mathrm{T}_{\mathrm{b}}\right)$ decides the length of the duration of tone signal

Specifically, we can express $g(t)$ in one period as:

$$
\begin{aligned}
& \mathrm{g}(\mathrm{t})=1 \quad 0 \leq \mathrm{t}<\mathrm{T}_{\mathrm{a}} \\
& 0 \quad \mathrm{~T}_{\mathrm{a}} \leq \mathrm{t}<\mathrm{T}_{\mathrm{p}}
\end{aligned}
$$


In a network, all the mobile nodes use the same data channel for packet transmission. The states of the data channel include:

- Receive: Node is receiving data packets from another node

- Send: The node is sending processed data to another node

- Idle: No packet is being received or transmitted and the data channel is free

- Collision: More than two nodes are using the data channel to transmit data at the same time, causing packet collision

The status of the data channel can be expressed by different pulse intervals using (1).

When a node $\mathrm{n}$ has packets to send, it first turns on its tone radio, sensing the channel to see whether it is free or not. It the state is received or collision, then $\mathrm{T}(\mathrm{t})=-\mathrm{T}(\mathrm{t})$ If the state is idle, then it is positive.

\section{Algorithm:}

If node $\mathrm{N}_{\mathrm{i}}$ has packets to send, then

If $\mathrm{T}(\mathrm{t})<0$, then Else

Keep monitoring the channel

If $\mathrm{T}(\mathrm{t})<\mathrm{SNR}$ or $\mathrm{S}<\mathrm{S}_{\min }$, then

Store the packets in a buffer B

Back off for random period $2^{\text {i-1 }} \mathrm{Xrand}(0,1) \mathrm{XCW}$ Else

If $\mathrm{T}(\mathrm{t})>=\mathrm{SNR}$ and $\mathrm{S}>=\mathrm{S}_{\min }$, then

Send the packet

where, $S_{\min }$ is the minimum threshold value for combined score of residual bandwidth and energy and where rand $(0,1)$ is a random number generator that generates a number evenly distributed between $(0,1)$, i is the number of times this packet has been retransmitted and $\mathrm{CW}$ is the contention window size.

Fair scheduling and queuing algorithm: The process of buffering packets until the channel threshold constraint is satisfied, is applicable only for nodes with better link quality, since they can always get the most bandwidth shares. As a result of this, the nodes with bad link quality has to wait until its channel quality recovers, leading to starvation. This unfairness problem can cause serious problem for the nodes with link quality less than packet overflow and long queuing delay.

A natural solution to this starvation problem is to adjust the link quality threshold value adaptively, depending on the current traffic load and queue length of the buffer.

To achieve energy efficient utilization while reducing the buffer overflow, we design a distributed adaptive threshold adjustment scheme. The scheme is detailed in this manner:

Buffer overflow can be prevented by predicting the future traffic load. This can be achieved by constantly measuring the queue length and its variation:

Initially Let $\mathrm{S}=\mathrm{S}_{\min }$

Let $\left\{\mathrm{t}_{\mathrm{i}}, \mathrm{t}_{\mathrm{i}+1}, \mathrm{t}_{\mathrm{i}+2}, \cdots \cdots\right\}$ denotes the sequence of packet arrival times of a node $n_{i}$

Let $Q\left(t_{i}\right)$ denote the queue length of the buffer of $n_{i}$ at time $\mathrm{t}_{\mathrm{i}}$

Then, $\left\{\mathrm{Q}\left(\mathrm{t}_{\mathrm{i}}\right), \mathrm{Q}\left(\mathrm{t}_{\mathrm{i}+\mathrm{k}}\right), \mathrm{Q}\left(\mathrm{t}_{\mathrm{i}+2 \mathrm{k}}\right), \ldots \ldots\right\}$ is the sequence of queue lengths at time instants $\left\{\mathrm{t}_{\mathrm{i}}, \mathrm{t}_{\mathrm{i}+\mathrm{k}}, \mathrm{t}_{\mathrm{i}+2 \mathrm{k}}, \ldots \ldots ..\right\}$ where $\mathrm{k}$ is the sampling interval for incoming packets.

Then the queue length variation can be calculated as:

$\mathrm{B}\left(\mathrm{t}_{\mathrm{i}+\mathrm{k}}\right)=\mathrm{Q}\left(\mathrm{t}_{\mathrm{i}+\mathrm{k}}\right)-\mathrm{Q}\left(\mathrm{t}_{\mathrm{i}}\right)$

$\Delta \mathrm{B}=\mathrm{B}\left(\mathrm{t}_{\mathrm{i}+2 \mathrm{k}}\right)-\mathrm{B}\left(\mathrm{t}_{\mathrm{i}+\mathrm{k}}\right)$

where, $\Delta \mathrm{B}$ is the prediction of queue variation at time $t_{i+2 k}$.

If $\Delta \mathrm{B}>=0$, the queue length has an increasing tendency; otherwise, if $\Delta \mathrm{B}<0$, the queue length is likely to decrease.

Based on the queue length variation prediction, we can develop a threshold adjustment scheme. We keep monitoring the incoming traffic and once the queue length exceeds a value $\mathrm{Q}_{\max }$ the threshold adjustment mechanism is started up.

\section{Algorithm:}

For each packet arrival at time $t_{i+k}$

Find $\mathrm{B}\left(\mathrm{t}_{\mathrm{i}+\mathrm{k}}\right)=\mathrm{Q}\left(\mathrm{t}_{\mathrm{i}+\mathrm{k}}\right)-\mathrm{Q}\left(\mathrm{t}_{\mathrm{i}}\right)$

If $\mathrm{Q}\left(\mathrm{t}_{\mathrm{i}+\mathrm{k}}\right) \mathrm{Q}_{\max }$ Then Find $\Delta \mathrm{B}>=\mathrm{B}\left(\mathrm{t}_{\mathrm{i}+2 \mathrm{k}}\right)-\mathrm{B}\left(\mathrm{t}_{\mathrm{i}+\mathrm{k}}\right)$ If $\Delta \mathrm{B}>0$ Then

$S=S_{\min }-\lambda$, where, $\lambda$ is the scale factor

Else if $\Delta \mathrm{B}<0$ and $\mathrm{S}<\mathrm{S}_{\min }$, Then $\mathrm{S}=\mathrm{S}+\lambda$

\section{End if}

End if

End for 
Performance evaluation: Simulation Model and Parameters: We use NS2 to simulate our proposed algorithm. In our simulation, the channel capacity of mobile hosts is set to the same value: $2 \mathrm{Mbps}$. In our simulation, 50 mobile nodes move in a $1000 \times 1000 \mathrm{~m}$ rectangular region for $100 \mathrm{sec}$ simulation time. Initial locations and movements of the nodes are obtained using the random waypoint (RWP) model of NS2. We assume each node moves independently with the same average speed. All nodes have the same transmission range of $250 \mathrm{~m}$. In this mobility model, a node randomly selects a destination from the physical terrain. It moves in the direction of the destination in a speed uniformly chosen between the minimal speed and maximal speed. After it reaches its destination, the node stays there for a pause time and then moves again. In our simulation, the speed is $10 \mathrm{~m} \mathrm{sec}^{-1}$. and pause time is $10 \mathrm{sec}$. The simulated traffic is Constant Bit Rate (CBR). For each scenario, ten runs with different random seeds were conducted and the results were averaged.

Performance metrics: We compare our proposed CAEFS MAC protocol with our previous EACA MAC protocol $^{[2]}$ and the standard IEEE 802.11 MAC protocol. We mainly evaluate the performance according to the following metrics:

- Aggregated Throughput: We measure aggregated throughput of all flows

- Average Energy Consumption: The average energy consumed by the nodes in receiving and sending the packets are measured

- Fairness Index: For each CBR flow, we measure the fairness index as the ratio of throughput of each flow and total no. of flows

\section{RESULTS}

The performance results of our proposed CAEFS MAC protocol with the effect of varying nodes and varying rates are shown below.

Effect of varying nodes: In the first experiment, we vary the number of nodes as 25, 50, 75 and 100 .

Initially we measure the energy consumption. Figure 1 shows that CAEFS consumes less energy than 802.11 and EACA when the network size is increased.

Next we measure the fairness index. Figure 2 shows that CAEFS achieves high fairness than 802.11 and EACA, when the network size is increased.

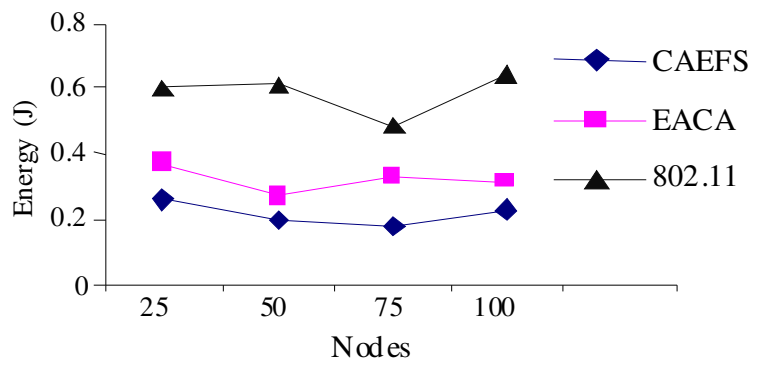

Fig. 1: Nodes Vs Consumed Energy

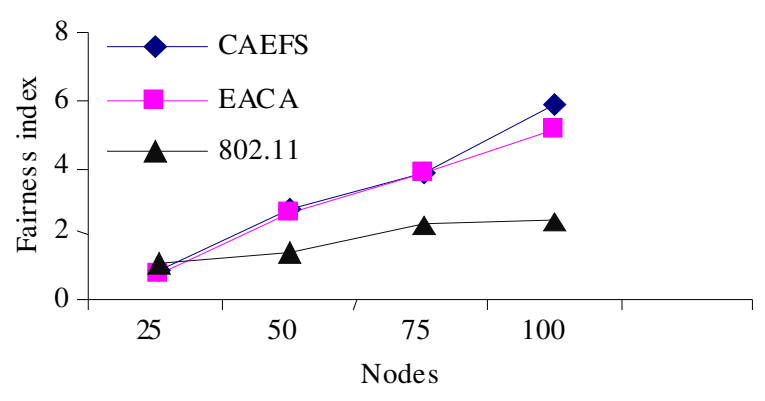

Fig. 2: Nodes Vs Fairness

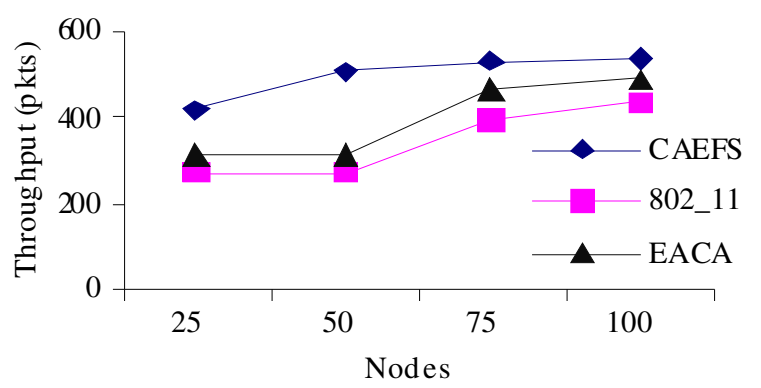

Fig. 3: Nodes Vs Throughput

The throughput against the number of nodes is calculated for all the 3 protocols and the results are shown in Fig. 3. From Fig. 3, we can see that the aggregated throughput of CAEFS is more when compared to 802.11 and EACA.

Effect of varying rates: In our second experiment, the packet sending rate is varied from $0.1-0.5 \mathrm{Mb}$.

Figure 4 shows the energy consumption of the nodes. From Fig. 4, we can see that CAEFS consumes less energy than 802.11 and EACA when the rate is increased.

Figure 5 shows that CAEFS achieves high fairness than 802.11 and EACA, when the rate is increased.

From Fig. 6, we can see that the aggregated throughput of CAEFS is more when compared to 802.11 and EACA. 


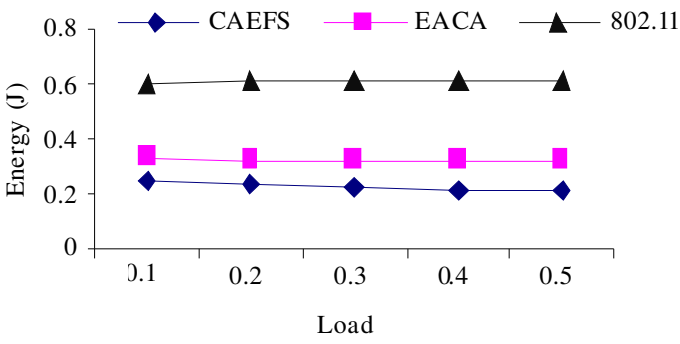

Fig. 4: Rate Vs Energy Consumption

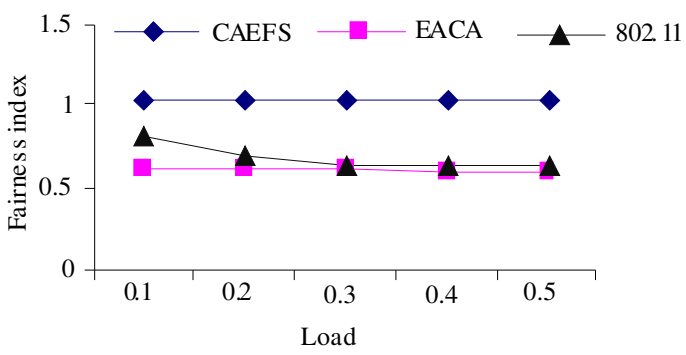

Fig. 5: Rate Vs Fairness

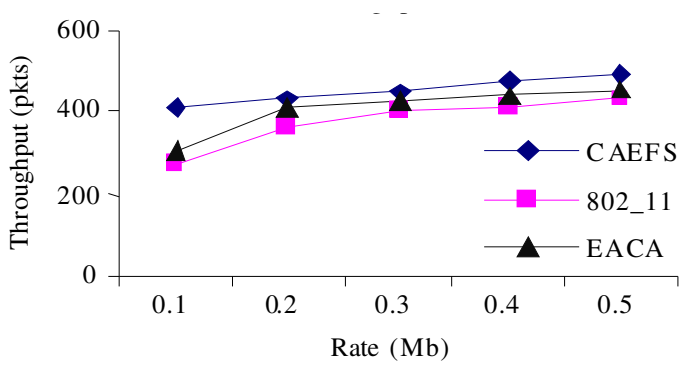

Fig. 6: Rate Vs Throughput

\section{DISCUSSION}

Since CAEFS transmits data through good quality links only, the average energy consumption will be less, compared with EACA. From ${ }^{[2]}$, we can observe that, EACA consumes less energy when compared to 802.11.

Because of its fair scheduling and queuing algorithm, CAEFS fairness will be slightly more than that of EACA and significantly higher than 802.11.

The average throughput generally depends on the link quality and channel state. Since CAEFS transmits flows with good link quality and channel condition, it yields very high average throughput.

\section{CONCLUSION}

In this study, we have developed a channel adaptive energy efficient MAC protocol, for efficient packets scheduling and queuing in an adhoc network, with time varying characteristic of wireless channel taken into consideration. In our proposed scheme, every node estimates the channel and link quality for each contending flow based on which a weight value is calculated and propagated using the routing protocol. Since a wireless link with worse channel quality can result in more energy expenditure, the transmission is allowed only for those flows whose weight is greater than minimum quality threshold. For flows with weight less than minimum quality threshold, the packets are buffered until the channel and link quality recovers or the weight becomes greater than the threshold. To avoid buffer overflow and achieve fairness for the poor quality nodes, we have also designed a fair scheduling and queuing algorithm. In our algorithm, we adaptively adjust the minimum quality threshold value, based on the current incoming traffic load variations. Through, simulation results, we have shown that, our proposed MAC protocol can achieve substantial energy savings with better fairness and increased throughput.

\section{REFERENCES}

1. Lin, X.H. and Y.K. Kwok, 2005. On channel adaptive energy management in wireless sensor networks. Proceeding of the International Conference Workshops on Parallel Processing, June 14-17, IEEE Computer Society, Washington DC., USA., pp: 397-404. DOI: 10.1109/ICPPW.2005.61

2. Priakanth, P. and P. Thangaraj, 2008. Energy and channel aware MAC protocol to achieve fairness in multi-hop mobile adhoc networks. Int. J. Comput. Sci. Network Secur., 8: 29-35. http://paper.ijcsns.org/07_book/200804/20080405.pdf

3. Chen, L. and W.B. Heinzelman, 2005. QoS-aware routing based on bandwidth estimation for mobile ad hoc networks. IEEE J. Select. Areas Commun., 23: 561-572. DOI: 10.1109/JSAC.2004.842560

4. Perkins, C.E. and E.M. Royer, 1999. Ad hoc on demand distance vector routing. Proceedings of the 2nd IEEE Workshop on Mobile Computing Systems and Applications, Feb. 25-26, New Orleans, LA., USA., pp: 90-100. DOI: 10.1109/MCSA.1999.749281

5. Padhye, J., S. Agarwal, V.N. Padmanabhan, L. Qiu, A. Rao and B. Zill, 2005. Estimation of link interference in static multi-hop wireless networks. Proceedings of the 5th ACM SIGCOMM Conference on Internet Measurement, Oct. 19-21, USENIX Association, Berkeley, CA., pp: 28-28. http://portal.acm.org/citation.cfm?id=1251086.1251114\# 
6. Ming, Y., J. Timoney, L. Doyle and D. O'Mahony, 2001. Evaluation of channel fairness models for ad-hoc networks. Proceedings of the 1st Joint IEI/IEE Symposium on Telecommunications Systems Research, Nov. 27-27, Dublin, pp: 4. https://www.cs.tcd.ie/ omahony/chan-fair.pdf

7. Kone, V., S. Das, B.Y. Zhao and H. Zheng, 2007. QUORUM: Quality of service in wireless mesh networks. Mobile Networks Applied, 12: 358-369. DOI: 10.1007/s11036-008-0050-8

8. Vo, H.Q. and C.S. Hong, 2008. Link quality-aware packet forwarding architecture for wireless mesh network. Proceeding of the 10th International Conference on Advanced Communication Technology, Feb. 17-20, pp: 929-932. DOI: 10.1109/ICACT.2008.4493919
9. Cheng, X., P. Mohapatra, S.J. Lee and S. Banerjee, 2008. MARIA: Interference-aware admission control and QoS routing in wireless mesh networks. Proceeding of the IEEE International Conference on Communications, May 19-23, IEEE Xplore Press, Beijing, pp: 2865-2870. DOI: 10.1109/ICC.2008.540

10. Chen, J., R. Lin, Y. Li and Y. Sun, 2008. LQER: A link quality estimation based routing for wireless sensor networks. Sensors, 8: 1025-1038. http://www.mdpi.org/sensors/ papers/s8021025.pdf 